\title{
Lubrication effects on the flow of wet granular materials
}

\author{
Qing $\mathrm{Xu}$, Ashish V. Orpe, and Arshad Kudrolli \\ Department of Physics, Clark University, Worcester, Massachusetts 01610
}

(Dated: October 29, 2018)

\begin{abstract}
We investigate the dynamics of a partially saturated grain-liquid mixture with a rotating drum apparatus. The drum is partially filled with the mixture and then rotated about its horizontal axis. We focus on the continous avalanching regime and measure the impact of volume fraction and viscosity of the liquid on the dynamic surface angle. The inclination angle of the surface is observed to increase sharply to a peak and then decrease as a function of liquid volume fraction. The height of the peak is observed to increase with rotation rate. For higher liquid volume fractions, the inclination angle of the surface can decrease with viscosity before increasing. The viscosity where the minima occurs decreases with the rotation rate of the drum. Limited measurements of the flow depth were made, and these were observed to show only fractional changes with volume fraction and rotation speeds. We show that the qualitative features of our observations can be understood by analyzing the effect of lubrication forces on the timescale over which particles come in contact.

PACS numbers: $45.70 . \mathrm{Ht}, 45.70 . \mathrm{Mg}$
\end{abstract}

\section{INTRODUCTION}

Granular flows have been studied over the last few decades in order to confront the challenges posed by several unresolved questions [1, 2, 3]. Most of these studies have focused on the relatively simple dry granular system with air as the interstitial fluid. However, lately, there has been a keen interest in understanding the dynamics of granular flows wetted by a small amount of liquid considering their prevalence in industry and nature [4, 5]. The addition of a small amount of liquid to a dry granular system results in the formation of liquid bridges which binds the particles. The static behavior of these liquid bridges has been studied for quite some time [6, 7] on the grain scale and the cohesive force generated is shown to be dependent on the surface tension of the liquid and grain size. On the macroscopic scale, there are a few quantitative descriptions of the impact of the liquid on the overall behavior. A wet pile of granular material is more stable than a dry pile [8, 9] and the failure in a wet pile occurs in the bulk rather than at the surface as for the dry case [10, 11]. Ratholing and jamming can occur during hopper drainage under conditions where dry grains would normally flow. Furthermore, particle segregation can be suppressed or enhanced by making them cohesive [12, 13, 14, 15]. Bond number (Bo) which is given by the ratio of the strength of the capillary bridge to the weight of a grain has been introduced as a useful measure of the cohesivity of the material [16].

An important system to study granular matter is a partially filled horizontally rotated drum. This system has been extensively used to study stability, avalanching, mixing and segregation properties of dry materials [17, $18,19,20,21,22,23]$. The flow is mostly confined to the surface and is only a few layers deep. A transition from stick-slip to continuous avalanching is observed as the rotation rate is increased. More recently, the rotating drum apparatus is being used to investigate wet granular materials because it offers many important features [11,
16, 24, 25, 26]. First, because the system is periodic, it can be investigated over long times in a steady state. Second, by simply varying the rotation rate, various timescales can be accessed. An attractive practical feature is that the system can be sealed very easily, which prevents contamination and evaporation of the liquids.

Recently, using this system, some of the static properties of a wet pile has been well characterized. Measurements of the angle of maximum stability as a function of the volume fraction of the liquid, the grain size, and the dimensions of the wet pile inside the drum have been made [26]. Two different models have been developed to describe the observed dependence. The first phenomenological approach considers the internal friction of the grains and models the increased stability due to increase in cohesive force [27, 28]. The second approach considers the geometric stability of the grains and the increases stability due to the orientation of the capillary bridges [26]. Both approaches yield similar scalings for the maximum stability in terms of the liquid and grain properties which are observed to be in good agreement with experimental observations [26].

Tegzes et al [24, 25] have performed detailed measurements of wet granular avalanches in the stick slip regime. They found that the nature of the avalanches depended on the volume fraction of the added liquid. The three flow regimes: granular, correlated, and viscoplastic were characterized by the nature of the stick-slip avalanches. Recently, simulations have considered the effect of adding simple cohesive forces on granular flow [29, 30].

In this paper, we discuss our investigation of the effect of liquids in the continous avalanche regime. We measure the angle of inclination as a function of rotation rate of the drum, and the volume fraction and viscosity of the added liquid. A wide drum is used to minimize the effect of the side walls. We define a lubrication number which is given by the ratio of the time needed for the two particles in neighbouring streamlines to move towards each other to the time needed to pass each other without contact. 


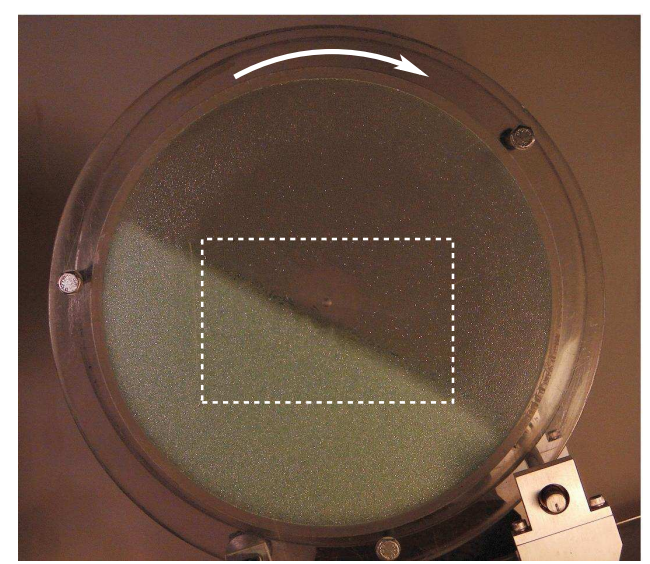

FIG. 1: An image of the experimental apparatus. The drum is partially filled with a mixture of glass beads and a small amount of silicone oil and rotated about its horizontal axis. The dotted box shows the region captured in the image to measure the surface angles.

This lubrication number is found to be useful in describing the observed variations of the inclination angles.

\section{EXPERIMENTAL APPARATUS}

The experimental apparatus consists of a clear plexiglass drum of diameter $28.5 \mathrm{~cm}$ and width $W=14.5 \mathrm{~cm}$ rotated about its horizontal axis as shown in Fig. 1. The drum is rotated using a computer controlled stepper motor allowing for a rotation rate $(\omega)$ variation from 0.00456 $\mathrm{rpm}$ to $13.69 \mathrm{rpm}$. The drum is $40 \%$ filled with spherical glass beads of diameter $d=1 \mathrm{~mm}$, premixed with small amount of viscous liquid. The amount of liquid added is reported in terms of volume fraction $V_{f}$, defined as the ratio of the volume of liquid to the volume of dry glass beads. The volume of dry beads is calculated as the weight of the beads divided by the bead density $\left(\rho_{b}=2.5\right.$ $\left.\mathrm{g} \mathrm{cm}^{-3}\right)$. The liquid used in the experiments is Silicone oil with four different viscosities $(\nu=0.05,0.2,1.0$ and $\left.10.0 \mathrm{~cm}^{2} \mathrm{~s}^{-1}\right)$.

Images are taken from one end of the drum and in the central region as indicated by a dotted box in Fig. 1. (The image resolution of $1024 \times 768$ pixels corresponds to a region of $18 \mathrm{~cm} \times 13.5 \mathrm{~cm}$ ). Images are captured using back lighting to identify the flowing surface. We have found that such a lighting scheme is less sensitive to a thin layer of grains which stick to side walls of the drum and therefore capture the surface of the granular mixture away from the side walls. The region with grains appear black in the image and unfilled regions of the drum appear white due to the back light. The free surface profile is extracted using an edge detection algorithm from which the angle of the surface is obtained. Measurements are made for the steady flow achieved after initial few revolutions of the drum. The reported values for the surface angle are obtained by averaging over 500 images (several revolutions of the drum). During the measurements, the drum is rotating continuously and particles are always in motion so that the particle-liquid mixture remains homogeneous throughout. The surface flow of the particles exhibits a stick-slip motion at lower rotational rates which changes to continuous motion at higher rotational rates. In the remainder of the paper, we discuss the flow behavior in the continuous avalanching regime.

\section{RESULTS AND DISCUSSION}

We first discuss the variation in the shape of the flowing surface with changing rotational speeds and the dependence of surface angles properties on the choice of location along the flowing surface where the measurements are made. The effect of interstitial liquid volume fraction and viscosity on the surface inclination is qualitatively discussed next. We then examine the relative effect of the liquid induced forces for various experimental parameters in order to explain the observed flow behavior.

\section{A. Inclination angle of the flowing surface}

The surface of the pile is almost linear at low $\omega$ and becomes progressively non-linear for higher $\omega$. Figure 2(a) shows a typical surface profile at low and high $\omega$ for wet systems. The non-linearity of the surface at higher $\omega$ results in different slopes along the surface. Figure 2(b) shows the slopes of the surface in the uppermost and lowermost portion of the extracted surface profile. The slopes (surface angles) are obtained by averaging over a region in which the profile is approximately linear. The surface angle of the upper section increases with $\omega$ while that in the lower section decreases slightly with $\omega$. This behavior is observed for dry case as well and the wet case for all the viscosities and volume fractions of the liquids investigated. Within the range of experimental parameters studied, the higher surface angle corresponding to the upper section appears to be the most sensitive to the variation in system parameters and in further discussion we focus on this angle to examine the effect of volume fraction and viscosity of the liquid added to particles.

\section{B. Effect of liquid volume fraction and viscosity}

Figure 3 shows the surface angle $(\theta)$ plotted against the rotational speed for different volume fractions $\left(V_{f}\right)$ of the added liquid of viscosity $\nu=1.0 \mathrm{~cm}^{2} \mathrm{~s}^{-1}$. Also shown is the data for the dry granular system (filled symbols) for the entire range of rotational rates studied. For all the cases studied, the surface angle increases with $\omega$, but the rate of increase progressively reduces with increasing amounts of liquid added. Notice particularly the 

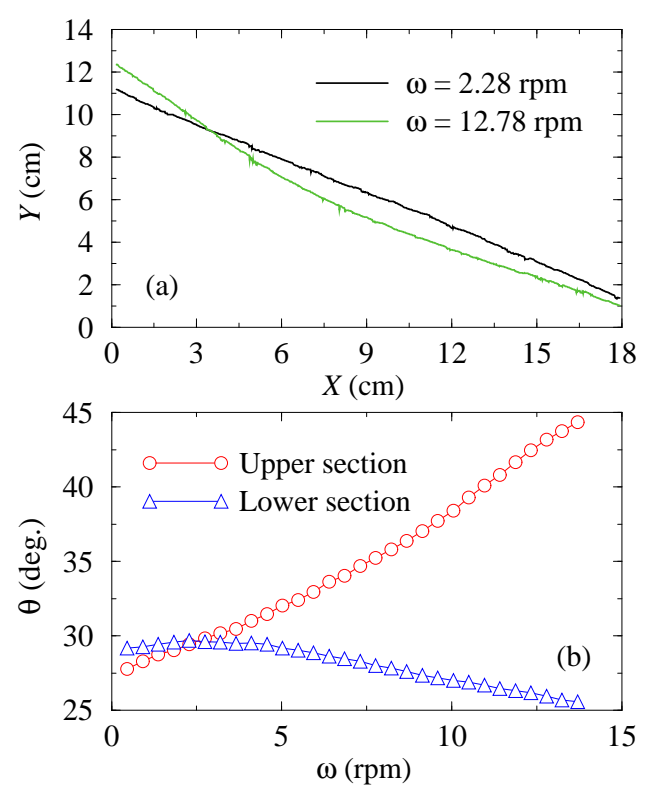

FIG. 2: (a) Free surface profile of the flowing particles as obtained from the images for two different rotational rates. (b) The surface angles calculated from upper and lower sections of the the free surface as a function of rotation rate. The data is obtained for the wet system with the added liquid of viscosity $\left(\nu=1.0 \mathrm{~cm}^{2} \mathrm{~s}^{-1}\right)$ and volume fraction $\left(V_{f}=0.48 \times 10^{-3}\right)$.

nearly flattened profile at the highest $V_{f}$. This rate of increase with rotational speed for all the volume fractions is slower than the quadratic dependence observed for the dry systems [17]. The added liquid tends to bind the particles strongly resulting in lower surface angles and slower rate of increase with rotational speeds. A small amount of liquid added $\left(V_{f}=0.08 \times 10^{-3}\right)$ shows a marginal, though a curious change. The angles for the dry system are higher than the wet system at lower rotational rate with the trend reversing at higher rotational rates. We attribute this behavior to the slight fluctuations in the angles for dry system due to humidity effects which were not controlled in the experiments. Further increase in the volume fractions results into higher surface angles than those for the dry case, suggesting the influence of the acting capillary attractive forces between particles thereby increasing the resistance to flow. However, adding more liquid also increases the lubrication between particles causing them to flow much easily as is seen for the highest volume fraction and higher rotational rates, where the angles observed are actually lower than those for the corresponding dry case. Similar behavior is observed for liquids with different viscosities.

To gain a further understanding about the effect of added liquid, we replot the data as shown in Figure 4 for four different viscosities studied. Consider first the case for $\nu=1.0 \mathrm{~cm}^{2} \mathrm{~s}^{-1}$. For lower rotational speeds, $\theta$ shows a rapid increase with volume fraction followed by a steady increase over the remaining range. The increase in the angle with volume fraction is a direct consequence

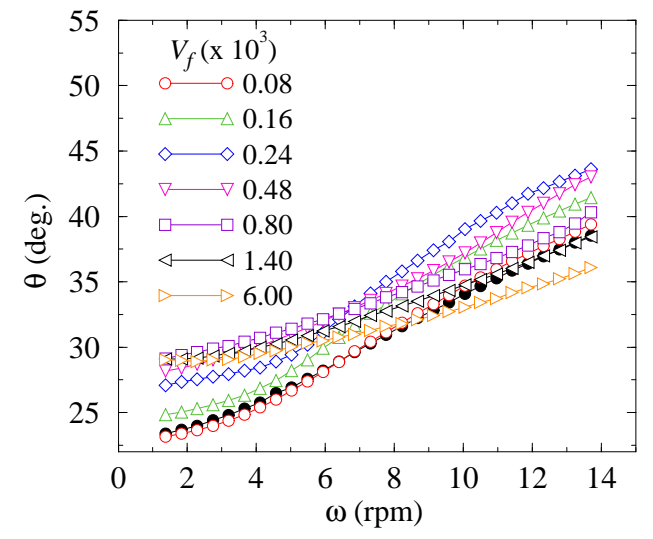

FIG. 3: (a) Variation of surface angle $(\theta)$ with the rotation rate for different volume fractions $\left(V_{f}\right)$ of the added liquid of viscosity $\nu=1.0 \mathrm{~cm}^{2} \mathrm{~s}^{-1}$. Filled circles denote the corresponding data for dry system.

of the cohesive capillary force due to the liquid bridges formed between particles as mentioned above. An important feature which is revealed is the formation of a peak in the surface angle profiles at higher rotational speeds. The angle rapidly increases to a maximum and then decreases continuously to a much lower value at higher volume fractions which is due to the flow being lubricated by the interstitial liquid. Similar trends are seen for different viscosities as well. The peak, however, is broad for lower viscosities and narrower for higher viscosities. A minimum observed at intermediate volume fractions and the highest rotational rate for $\nu=10.0 \mathrm{~cm}^{2} \mathrm{~s}^{-1}$ is not quite clear and suggests a complex interplay of the forces due to the liquid.

To further elucidate the effect of viscosity, we plot the surface angles versus the viscosity for different rotational speeds and for the highest volume fraction studied as shown in Figure 5. For lower rotational speeds the angles increase monotonically with viscosity which is expected considering that the resistance to flow would increase with viscosity. However, the angles show a reverse behavior at the highest rotational rates, wherein the liquid actually assists the flow and reduces the angles. A combination of these effects is seen at intermediate rotational rates wherein the angles decrease to a minumum and then increase for higher viscosities.

The discussion so far suggests that the effect of the added liquid is sensitive to the rotational rates employed. The primary effect of adding the liquid is to generate two newer forces between the particles viz. the attractive capillary forces and the repulsive viscous forces in addition to the gravity and fricitional forces which exist in a dry system. In the next section, we examine these forces and their relative dominance with respect to each other for different rotational rates which would help in understanding the observed behavior of the surface angles. 


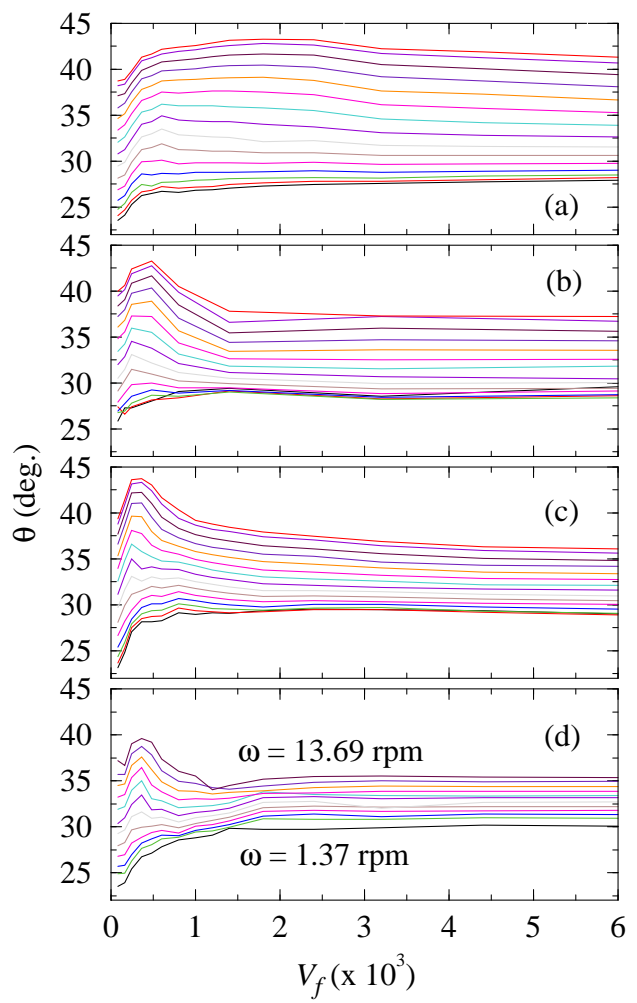

FIG. 4: Variation of the surface angle with volume fraction for different rotation rates. (a) $\nu=0.05 \mathrm{~cm}^{2} \mathrm{~s}^{-1}$, (b) (a) $\nu=0.2$ $\mathrm{cm}^{2} \mathrm{~s}^{-1}$, (c) $\nu=1.0 \mathrm{~cm}^{2} \mathrm{~s}^{-1}$ and (d) $\nu=10.0 \mathrm{~cm}^{2} \mathrm{~s}^{-1}$. In all cases, the rotational rates range from $1.37 \mathrm{rpm}$ to $13.69 \mathrm{rpm}$ as specified in (d).

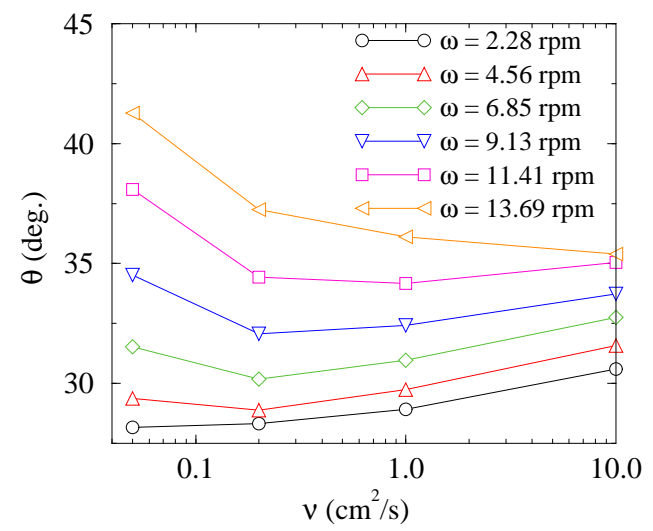

FIG. 5: Variation of the surface angle with viscosity for different rotational rates and fixed volume fraction $\left(V_{f}=6.0\right.$ $\left.\times 10^{-3}\right)$.

\section{Effect of liquid induced forces}

When a small amount of liquid is mixed with dry beads, the liquid wets the bead system and forms liquid bridges between the particles bonding them together by

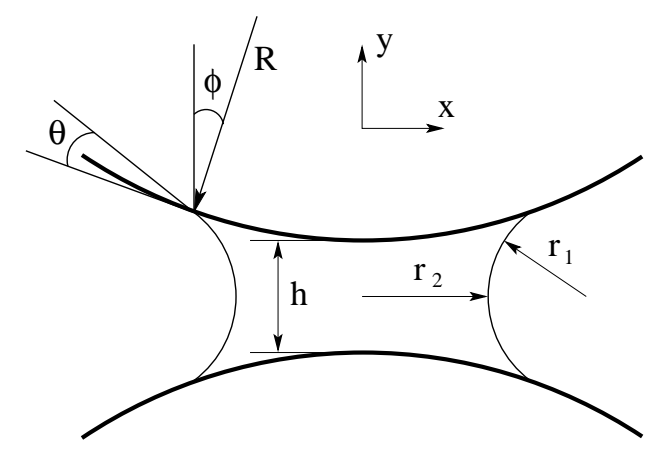

FIG. 6: Schematic of a liquid bridge formation between two spherical particles. $\theta$ is the liquid/solid contact angle, $\phi$ is the half-filling angle, $R=(d / 2)$ is the diameter of the particle and $h$ is the distance between two grains. $r_{1}$ and $r_{2}$ are respectively the radius of the neck and the meridian profile.

a weak attractive capillary force. A schematic of such a liquid bridge between two particles is shown in Figure 6 . In the limit of small amount of liquid, the gravity effects can be neglected and the total attractive force can be expressed as a sum of the component of surface tension $(\sigma)$ force along the length of the bridge and the force arising due to the pressure difference between air and liquid. Further, assuming $d / 2 \gg r_{2} \gg r_{1}$ and $h \ll 2 r_{1} \cos \theta$ and that the liquid bridge is approximately cylindrical (flat profile), a simplified expression can be obtained relating the total force to the bridge volume $(V)$ and separation distance $(h)$ which is given as 31]

$$
F_{\text {cap }} \approx \pi d \sigma \cos \theta\left[1-\frac{1}{\sqrt{1+\frac{4 V}{\pi d h^{2}}}}\right] .
$$

where $V=\pi d\left[H^{2}(b)-h^{2}\right] / 4, H(r)=h+2 r^{2} / d, r$ is the radial distance from the center of the bridge and $b$ is the radius of the wetted area. The capillary force varies directly as the volume of the liquid bridge which depends on the volume fraction of added liquid.

The pressure change in the liquid bridge arises due to the relative displacements of two particles with respect to each other while in flow and the two can be related by the Reynolds equation which can be simplified to determine the expression for the viscous force acting on the particles. For finite volumes of liquid (cylindrical bridge) the viscous force is given as 31]

$$
F_{v i s}=\frac{3}{8} \pi \rho_{l} \nu d^{2}\left[1-\frac{h}{H(b)}\right]^{2} \frac{1}{h} \frac{d h}{d t} .
$$

where $\rho_{l}$ is the density of the liquid, $d h / d t$ is the rate of approach of particles towards each other. $H(b)$ is calculated from the expression given above. The viscous force varies directly as the rate of displacement of particles, is negligible for large separation distances and diverges as particles come very close to each other.

Direct measurments or estimates of these liquid induced forces in addition to the gravity and frictional 


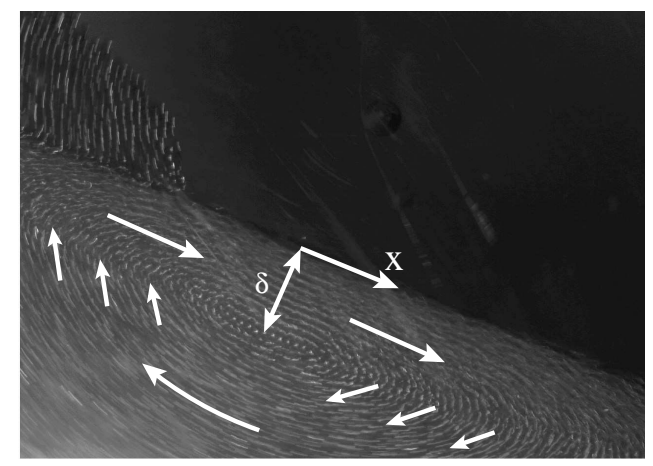

FIG. 7: An image taken with 1/4 second exposure shows the depth of the surface flow. Volume fraction $V_{f}=0.08 \times 10^{-3}$, rotation rate $\omega$ is $2.28 \mathrm{rpm}$. The arrows indicate the general direction of the flowing particles.

forces for various experimental parameters would elucidate the observed behavior of surface angles. However, our experimental techniqe prevents the direct estimates of these forces between the particles. So instead, we examine different scenarios determining the relative significance of different forces with repect to each other. This can be done by calculating the appropriate timescales corresponding to the motion of particles relative to each other which can be obtained through velocity profiles in the flowing layer of particles. The spreading of the interstitial liquid on the end plates combined with the sticking of the particles to the walls prevents accurate measurements of the velocities in the flowing layer. To get around this, we instead measure the flowing layer thickness $(\delta)$ using streakline photography similar to that used earlier 21] for the dry systems. This involves taking time exposed photographs to obtain particle streaklines as shown in Fig. 7 Because we are seeking estimates, we focus on the central flow region and calculate the maximum layer thickness over there. We note here that the problems faced while measuring accurate particle velocities do not hinder the measurements of the flowing layer thickness. The typical flow direction of particles and the location for layer thickness $(\delta)$ measurement is shown in Fig. 7.

The variation of the flowing layer thicknes with rotational rate $(\omega)$ for different volume fractions and for one particular viscosity of the liquid is shown in Fig. 8. The layer thickness changes very slowly with rotational rate, which is similar to the results for the dry systems with the measurements using NMR [32, 33] and particles visualization [21] techniques. The rate of change decreases with increasing rotational rates, particularly for higher volume fractions with the layer thickness approaching a constant value at high enough rotational rates, similar to the trends observed for dry systems. Further, the layer thickness increases much slowly with rotational rates for higher volume fractions and interestingly the layer thickness at the highest volume fraction becomes almost constant and independent of the rotatational speed. Similar behavior is also observed for liquids with different viscosi-

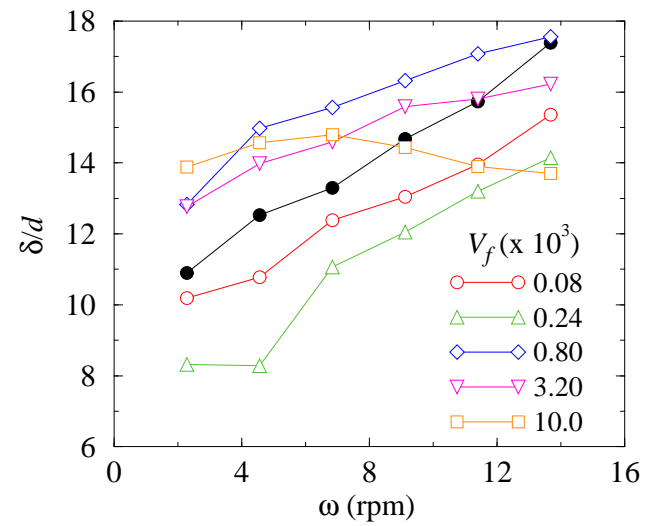

FIG. 8: Variation of the normalized flow depth $(\delta / d)$ with rotation rate for different volume fractions of the added liquid $\left(\nu=0.2 \mathrm{~cm}^{2} / \mathrm{s}\right)$, where $d$ is the diameter of the particle. Filled circles denote the corresponding data for dry system.

ties. For the steady flow of particles in the continuous flow regime, the flux $Q$ of the particles (at the central location where $\delta$ is measured) can be written from mass conservation as

$$
Q=\frac{1}{2} \rho_{b}\left(\omega z R_{0}\right) R_{0}=\rho_{b} V_{x} z \delta
$$

where $\rho_{b}$ is the density of glass beads (assumed to be approximately equal in the flowing layer and rotating bed), $R_{0}$ is the radius of the drum, $z$ is the length of the cylinder and $V_{x}$ is the average velocity in the flowing layer. The average velocity in the flowing layer can be then written as

$$
V_{x}=\frac{\omega R_{0}^{2}}{2 \delta} .
$$

Using Eq. 4, we can thus get the estimates of the representative velocities in the flowing layer for various rotation rates, volume fractions and viscosities of the interstitial liquid. For the particles flowing in the $x$-direction (see Figure 7), we denote $t_{x}=d /\left(V_{x}\right)$ as the time required for the two particles in the neighbouring streamlines to travel past each other, where $d$ is the particle diamete r. Substituting for $V_{x}$ from Eq. 4 gives

$$
t_{x}=\frac{2 d \delta}{\omega R_{0}^{2}}
$$

The timescale $t_{x}$ primarily varies inversely as the rotational rate $(\omega)$ since $\delta$ is only weakly dependent on $\omega$ and volume fractions as seen from Figure 8 ,

We now define $t_{y}$ as the timescale for the same two particles to approach each other while squeezing out the liquid from between. Balancing the viscous force between the two particles given by Eq. 2 to the weight of the particle $\left(\pi d^{3} \rho_{b} g / 6\right)$ and then integrating the resulting equation gives

$$
t_{y}=\frac{9}{4} \frac{\nu \rho_{l}}{d g \rho_{b}}\left[\frac{2 b^{2} d\left(h_{s}-h_{0}\right)}{\left(2 b^{2}+h_{s} d\right)\left(2 b^{2}+h_{0} d\right)}\right.
$$




$$
\left.+\ln \left(\frac{1+2 b^{2} / h_{s} d}{1+2 b^{2} / h_{0} d}\right)\right]
$$

where $h_{s}$ is the surface roughness of the beads, and for the glass beads is approximately $10^{-3} d$. $b$ is the radius of the wetted area of the particle. The above equation gives an estimate of the time required by the two particles separated by a distance $h_{0}$ to approach each other to a distance within surface roughness $\left(h_{s}\right)$.

The ratio $R a=t_{x} / t_{y}$, after substituting for the experimental parameters, can be then written as:

$$
R a=\frac{0.13 \delta /(\omega \nu)}{\left[\frac{2 b^{2} d\left(h_{s}-b\right)}{\left(2 b^{2}+h_{s} d\right)\left(2 b^{2}+b d\right)}+\ln \left(\frac{1+2 b^{2} / h_{s} d}{1+2 b / d}\right)\right]}
$$

where, we have assumed that $h_{0} \sim b$, i.e. liquid bridges just before or after snapoff are semi-spherical droplets on the surface of the beads. We can now investigate various scenarios under which different forces can be dominant. For $R a \ll 1$, the two particles pass each other without coming in contact (thus minimizing the frictional forces) while for $R a \gg 1$ the viscous forces between the particles dominate the overall flow behavior. In the following we now estimate these time scales for different rotational rates, volume fractions and viscosities of the liquids used in our experiments.

For small $V_{f}$, as when the beads first get uniformly coated with the liquid, and liquid bridges are formed between the grains, $b \sim h_{s}$ [27]. In that limit, both the terms in the denominator of Eq. (77) tend to 0 and therefore $R a \gg 1$ and particles touch as they flow past each other and kinetic friction/viscous forces become important resulting in the higher surface angles. This flow regime corresponds to the surface angles increase with volume fraction (see Fig. (4) leading to a maximum. The peak in the profile would in that case correspond to the maximum value of $R a$.

On the other hand, for large enough volume fractions, $b \sim d / 2, h_{0} \sim d / 2$ and using $h_{s} \sim 10^{-3} d$ and $\delta \sim 14 d$ (from Fig. 8), we get $R a \rightarrow 10^{-2} /(\omega \nu)$. For highest rotational rate $(\omega=13.69 \mathrm{rpm})$ and lowest viscosity $\left(\nu=0.05 \mathrm{~cm}^{2} \mathrm{~s}^{-} 1\right), R a \rightarrow O\left(10^{-1}\right)<1$. We thus expect the surface angles to be lower and is indeed seen from the decrease in surface angles with volume fraction (see Fig. 4(a)). This effect is more pronounced at the highest viscosity used $\left(\nu=10.0 \mathrm{~cm}^{2} \mathrm{~s}^{-1}\right)$ for which $R a \rightarrow O\left(10^{-3}\right) \ll 1$. Note the faster decay in the surface angles with increasing volume fractions in Fig. 4(c,d). The rotation rate and viscosity, thus, act together to give maximal lubrication. This is reflected in the non-intuitive decrease in the surface angles with increasing viscosity at highest rotational rate (see the topmost curve in Fig. 5).

The simple analysis presented here based on the time scales for the particles to come in contact serves to explain most of the effects due to varying volume fractions of the liquid for different rotational rates and viscosities. The primary dependence on the viscosity of the liquid or the rotational rates for a given volume fraction, however, cannot be explained using this analysis. For example, the angle increases with viscosity at lower rotational rates, the reason being the increase in the drag forces with viscosity leading to additional resistance to flow and hence higher angles (see the bottom curve in Fig. 5). Using Eq. (7) alone in this instance would cause the corresponding $R a$ values to change from $O(1)$ to $O\left(10^{-2}\right)$ suggesting a decrease in the angle contrary to that observed. A similar argument also holds true for the case where the angles increase with rotational rates (the corresponding $R a$ actually decreases in those instances). Apart from these behaviors, the analysis cannot as well account for the more complex behaviors like the sharp minimum in the surface angles observed at higher rotational rates and highest viscosity (see Fig. 4d) or the decrease in the angle followed by an increase with increasing viscosity observed for intermediate rotational rates (see Fig. 5). Understanding these complicated effects would require a more rigorous study involving estimation of the different competing forces which is beyond the scope of this work.

\section{SUMMARY}

In summary, we have investigated the effect of volume fraction, viscosity of the liquid, and rotation rate of the drum on the inclination angle of a glass bead pile in a rotating drum. The surface angle show a non-linear increase with the rotational rate for any particular liquid volume fraction and viscosity. For any rotation rate and liquid viscosity, adding small amounts of liquid increases the surface angle than the corresponding values for the dry system which is a direct consequence of the liquid bridge formation between particles thus increasing the resistance to flow. However, continuing the addition of liquid eventually leads to a constant slow increase in the surface angles at low rotational rates whereas the angles show a surprising decrease at high enough rotation rates even though the strength of liquid bridge does not decrease. The effect is seen to be more dramatic for higher viscosities. By doing an analysis of the time scales related to the approach of two particles towards each other during flow using we derived a criterion for determining the relative effects of friction and lubrication between flowing particles. The analysis is valid primarily for accounting the effects due to varying liquid volume fractions and captures most of the observed variation in the angle of inclination. However, we are unable to make simple comparisions of the scaling because of different competing forces in the experiments which are not measurable using the techniquies employed here. Additionally, it would be helpful to have detailed velocity profiles and flow depths away from the sidewalls as a function of liquid content, and will be the focus of future work. 


\section{ACKNOWLEDGMENTS}

We thank J. Norton for his help with building the apparatus, and J. Xia and H. Wang for helpful discussions.
This work was supported by the National Science Foundation grant number CTS-0334587 and the donors of the Petroleum Research Fund.
[1] "Granular solids, liquids, and gases," H. M. Jaeger, S. R. Nagel, and R. P. Behringer, Rev. Mod. Phys. 68, 1259 (1996).

[2] "Granular Matter: a tentative view," P. G. deGennes, Rev. Mod. Phys. 71, S374 (1999).

[3] "Granular flows," J. Rajchenbach, Advances in Physics, 49, 229 (2000).

[4] "Dynamics of wet granular matter," S. Herminghaus, Adv. Phys., 54, 221 (2005).

[5] "Wet granular materials," N Mitarai and F Nori, Adv. Phys., 55 1-45 (2006).

[6] "Tensile Strength of Wet Granular Materials," G. Mason and W. C. Clark, Chemical Engineering Science 20, 859 (1965).

[7] "A theoretical study of liquid bridge forces and stability between two rigid spherical bodies," G. Lian, C. Thornton, and M. J. Adams, Journal of Colloid and Interface Science 161, 138 (1993).

[8] "What Keeps Sandcastles Standing," D. Hornbaker, R. Albert, I. Albert, A. Barabasi, and P. Schiffer, Nature 387, 765 (1997).

[9] "Moisture-induced ageing in granular media and the kinetics of capillary condensation," L. Bocquet, E. Charlaix, S. Ciliberto, and J. Crassous, Nature 396, 735 (1998).

[10] "How Sandcastles Fall," T. C. Halsey and A. J. Levine, Phys. Rev. Lett. 80, 3141 (1998).

[11] "Looking for Self-organized critical behavior in avalanches of slightly cohesive powders," M. A. S. Quintanilla, J. M. Valverde, A. Castellanos and R. E. Viturro, Phys. Rev. Lett. 87, 194301 (2001).

[12] "Segregation transitions in wet granular matter," A. Samadani and A. Kudrolli, Phys. Rev. Lett. 85, 5102 (2000).

[13] "Angle of repose and segregation of cohesive granular matter," A. Samadani and A. Kudrolli, Phys. Rev. E 64, 051301 (2001).

[14] "Controlling Cohesive Particle Mixing and Segregation," H.-M. Li and J. J. McCarthy, Phys. Rev. Lett. 90, 184301 (2003).

[15] "Phase diagrams for cohesive particle mixing and segregation," H.-M. Li and J. J. McCarthy, Phys. Rev. E 71, 021305 (2005).

[16] "Discrete characterization tools for cohesive granular material," S. T. Nase, W. L. Vargas, A. A. Abatan and J. J. McCarthy, Powder Technology, 116, 214 (1999).

[17] "Flow in powders: From discrete avalanches to continuous regime," J. Rajchenbach, Phys. Rev. Lett. 65, 2221 (1990).

[18] "Transverse flow and mixing of granular materials in a rotating cylinder," D. V. Khakhar, J. J. McCarthy, Troy
Shinbrot, and J. M. Ottino, Phys. Fluids 9, 31 (1997).

[19] "Continuous Avalanche Segregation of Granular Mixtures in Thin Rotating Drums," H. A. Makse, Phys. Rev. Lett. 83, 3186 (1999).

[20] "Mixing and segregation of granular materials," J.M. Ottino and D.V. Khakhar, Annu. Rev. Fluid Mech. 32, 55 (2000).

[21] "Scaling relations for granular flow in quasi-twodimensional rotating cylinders," A. V. Orpe and D. V. Khakhar, Phys. Rev. E 64, 031302 (2001).

[22] "Multiscale Clustering in Granular Surface Flows," D. Bonamy, F. Daviaud, L. Laurent, M. Bonetti, and J.-P. Bouchaud, Phys. Rev. Lett. 89, 034301 (2002).

[23] "Continuum theory of partially fluidized granular flows," I. S. Aranson and L. Tsimring, Phys. Rev. E 65, 061303 (2002).

[24] "Avalanche Dynamics in Wet Granular Materials," P. Tegzes, T. Vicsek, and P. Schiffer, Phys. Rev. Lett. 89, 094301 (2002).

[25] "Development of correlations in the dynamics of wet granular avalanches," P. Tegzes, T. Vicsek, and P. Schiffer, Phys. Rev. E 67, 051303 (2003).

[26] "Maximum angle of stability of a wet granular pile," S. A. Nowak, A. Samadani, and A. Kudrolli, Nature Physics 1, 50 (2005).

[27] "Critical angle of wet sandpiles," T. G. Mason, A. J. Levine, D. Ertas, and T. C. Halsey, Phys. Rev. E 60, R5044 (1999).

[28] "Where does a cohesive granular heap break?" F. Restagno, L. Bocquet and E. Charlaix, The European Physical Journal E 14, 177 (2004).

[29] "Discrete-particle simulations of cohesive granular flow using a square-well potential," M.W. Weber, D.K. Hoffman, and C.M. Hrenya, Granular Matter 6, 239-254 (2004).

[30] "Plug flow and the breakdown of Bagnold scaling in cohesive granular flows," R. Brewster, G. S. Grest, J. W. Landry, and A. J. Levine, Phys. Rev. E 72, 061301 (2005).

[31] "Liquid bridge between two moving spheres: An experimental study of viscosity effects," O. Pitois, P. Moucheront, and X. Chateau, J. Colloid and Interface Sci. 231, 26-31 (2000)

[32] "Non-invasive measurements of granular flows by magnetic resonance imaging," M. Nakagawa, S. A. Altobelli, A. Caprihan, E. Fukushima and E. -K. Jeong, Exp. Fluids, 16, 54 (1993)

[33] "Steady particulate flows in a horizontal rotating cylinder," K. Yamane, M. Nakagawa, S. A. Altobelli, T. Tanaka and Y. Tsuji, Phys. Fluids, 10, 1419 (1998) 